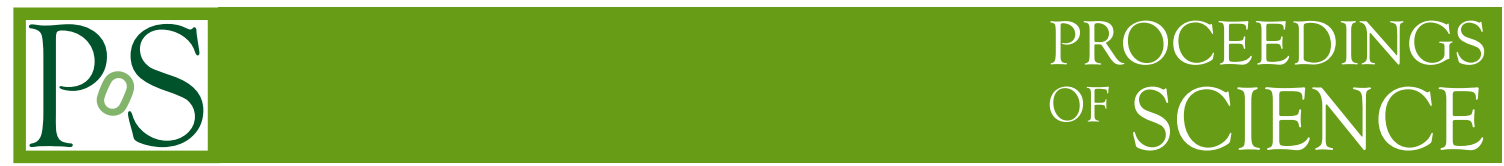

\title{
Overview of physics possibilities at future DIS facilities
}

\section{Anna M. Stasto*}

Department of Physics, The Pennsylvania State University, University Park, PA 16802, U.S.A.

E-mail: ams 52 apsu. edu

In this talk I will present a brief overview of the selected physics topics that can be explored at future electron-proton and electron-ion colliders: Electron Ion Collider (EIC) in the US, Large Hadron electron Collider (LHeC) and Future Circular Collider in electron-hadron option (FCCeh) at CERN.

XXVII International Workshop on Deep-Inelastic Scattering and Related Subjects - DIS2019 8-12 April, 2019

Torino, Italy

${ }^{*}$ Speaker. 


\section{Introduction}

Deep Inelastic Scattering is an excellent process to explore the structure of the proton. The highly energetic lepton, for example electron, scatters off the hadronic target - proton or a nucleus. In this way one can unravel the inner structure of the target with resolution controlled by the virtuality of the exchanged boson. The seminal DIS experiment by the MIT-SLAC group lead to the discovery of partons, the constituents of the hadrons. The next generation of DIS experiment was the HERA collider at DESY, Hamburg, the only electron(positron)-proton collider up to date, which reached center-of-mass energy of $\sqrt{s}=318 \mathrm{GeV}$. HERA established a rapid growth of the proton structure function $F_{2}$ with decreasing Bjorken $x$, which was subsequently understood as being driven by the increasing gluon density due to gluon splitting in QCD. HERA measurements of the structure function, and the extraction of the parton distribution functions are crucial for making the precise predictions for the other processes in hadron collisions, in particular at the LHC such as production of jets, heavy quarks and Higgs. It is also of great importance for the high energy neutrino astrophysics, explored for example at the neutrino observatory IceCube. The highly energetic neutrinos interact mainly through the DIS process at high energies, and therefore precision knowledge of the parton distribution functions at small values of Bjorken $x$ is essential.

Despite great success in establishing a new era in high energy deep inelastic scattering, HERA had some limitations, mainly due to the relatively low luminosity, lack of polarization and lack of nuclei as targets. Thus it is mandatory to plan the future DIS facility which could explore the high intensity and high energy frontier in structure of nucleons, and also providing valuable information on the structure of nuclei which is essential for understanding the initial state in heavy ion collisions.

In this presentation I shall briefly review physics possibilities at three machines: Electron Ion Collider in the US [1], Large Hadron electron Collider [2] and Future circular Collider [3] in the electron-hadron option at CERN.

The Electron Ion Collider (EIC) is the proposed facility in the United States, which will collide electrons of energies $5-20 \mathrm{GeV}$ with protons and nuclei, to reach center of mass energy of around $20-140 \mathrm{GeV}$ depending on the configuration. The designed luminosity is $10^{34} \mathrm{~cm}^{-2} \mathrm{~s}^{-1}$. The EIC will have a polarization of electrons, protons, deuterons and ${ }^{3} \mathrm{He}$, and will be able to use wide range of nuclei as targets. Large Hadron electron Collider ( $\mathrm{LHeC})$ and Future Circular Collider in electron-hadron (FCC-eh) option are proposals for the DIS facility at CERN. In both cases the Energy Recovery Linac would accelerate electrons to the energies up to $60 \mathrm{GeV}$. The $\mathrm{LHeC}$ is the proposal to utilize proton and ion beams from the High Luminosity LHC whereas FCC-eh would use the beam from the hadronic FCC. The center of mass energies would be $1.3 \mathrm{TeV}$ and $3.5 \mathrm{TeV}$ for proton case, and $812 \mathrm{GeV}$ and $2.2 \mathrm{TeV}$ for lead beams for $\mathrm{LHeC}$ and FCC-eh respectively. The designed luminosity for both machines are $10^{34} \mathrm{~cm}^{-2} \mathrm{~s}^{-1}$. All the proposed machines have a rich complementary physics program: while the EIC would explore the spin structure of the proton, and provide information about the nuclear structure at intermediate energy for wide range of nuclei, the CERN proposals would offer precision measurements of parton distribution functions, precision top electroweak and measurements of Higgs couplings and nuclear structure for high energies. In Fig. 1 the $\left(x, Q^{2}\right)$ range is shown for all three machines, and compared with HERA and fixed target measurements of the proton structure. The EIC would allow to probe previously unexplored region 
in the proton spin structure. The $\mathrm{LHeC}$ and FCC-eh would extend the range in $x$ and in $Q^{2}$ in unpolarized DIS by orders of magnitude which will be crucial for new experiments and theory developments.

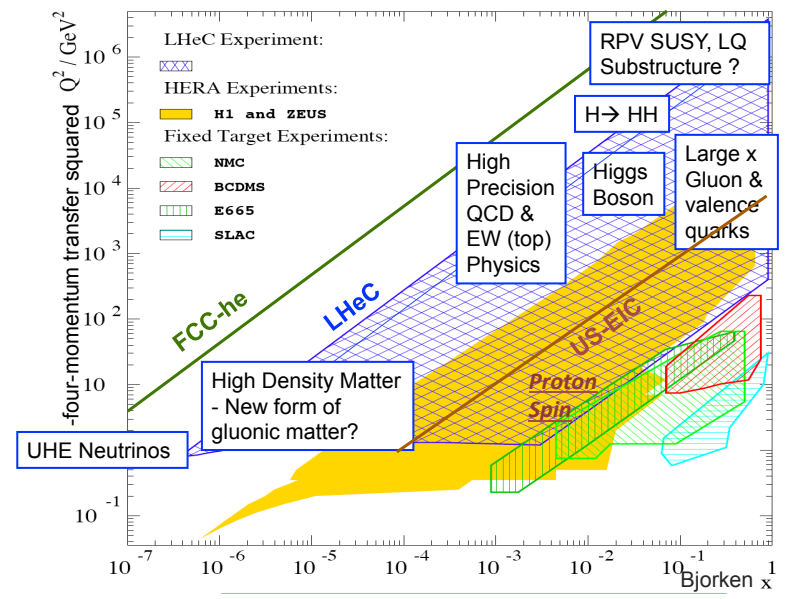

Figure 1: $\left(x, Q^{2}\right)$ kinematics for EIC, LHeC, FCC-eh compared with HERA and fixed target experiments.

\section{Parton distribution functions}

The LHeC and FCC-eh machines would offer unprecedented precision to measure the unpolarized parton distribution functions in the proton. Large lever arm in $Q^{2}$ and $x$, small systematic errors, and large luminosity will allow to extract very precisely the PDFs. The data will allow for the precise determination of the charm (to $3 \mathrm{MeV}$ ) and bottom mass (to $10 \mathrm{MeV}$ ) as well as $V_{c s}$ (to $1 \%$ ). The measurement of the strong coupling constant at the $\mathrm{LHeC}$ would be performed at the per mille level from DIS data. Of particular importance is the fact that such machines would be able to unfold for the first time completely the parton distribution functions in the proton through the $\mathrm{NC}$ and $\mathrm{CC}$ interactions as well as the heavy quark production. The high $Q^{2}$ region would be particularly interesting for studying the weak interactions since, $\mathrm{LHeC}$ and FCC-eh would allow to study the $W$ and $Z$ exchange dominated DIS, and therefore would be able to resolve the flavors, including $\mathrm{NC}$ and $\mathrm{CC}$ production of heavy quarks.

\section{Novel dynamics at low $x$}

Both LHeC and FCC-eh will offer unprecedented capabilities in studying the novel dynamics at very low values of Bjorken $x$. The seminal work of Balitsky-Fadin-Kuraev-Lipatov established the evolution equation which sums the very large logarithms of energy. The BFKL approach need to be however stabilized by the resummation, and procedures to achieve that have been established two decades ago and shown to substantially reduce the intercept which governs the energy growth of the cross sections which are dominated by the BFKL dynamics. The resummation schemes have been recently applied to the HERA data and showed marked improvement over the fixed order calculations. Since both $\mathrm{LHeC}$ and FCC-eh extend the range of $x$ at low and moderate values of $Q^{2}$, it is expected that the small $x$ effects will be much more prominent in these machines. 
Another phenomenon that can be studied at these machines is the parton saturation. It has been predicted that QCD exhibits parton saturation which occurs when the gluon density becomes sufficiently large and the two competing mechanisms, of gluon splitting and recombination, will start to balance each other. Simulations demonstrate that when saturation effects are present, standard fits based on the DGLAP evolution fail to describe the simulated LHeC data when $F_{2}$ and $F_{L}$ (of $F_{2}$ for charm) are simultaneously fitted. This can only be resolved with the LHeC of FCC-eh in an unambiguous way as it requires high precision DIS data in a kinematic range extended to sufficiently large values of $Q^{2}$ to ensure the dominance of the small coupling regime.

\section{Inclusive and exclusive diffraction}

HERA collider established that about $10 \%$ events in electron-protons collisions are diffractive, that is events which are characterized by the presence of the large rapidity gap, where in the detector there is a large region void of any particle activity. Such events are usually described within QCD by the exchange of a colorless object that carriers quantum number of a vacuum. The diffractive events can be either identified through the proton tagging or by large rapidity gap method. There is a plethora of different diffractive processes that can be investigated at a electron-proton and electron-ion colliders. The inclusive diffraction can be measured over a wider range of $x$ and $Q^{2}$ and parton distribution functions in diffraction can be extracted with a much better precision than at HERA. In this way one can test the theorems of factorization in diffraction by comparisons of calculations based on the diffractive PDF extraction from inclusive data and the diffractive production of heavy flavors and jets. The extended kinematics at LHeC and FCC-eh will allow for the production of diffractive top and $W$ and $Z$ bosons. By performing simultaneous measurements of the inclusive structure functions for scattering in nuclei a deep theoretical relation between shadowing and diffraction can be tested. In particular an Electron Ion Collider could test the shadowing on the deuteron, which has a clear theoretical relation to the inclusive diffraction on the proton.

The collinear parton distribution functions provide only information about the longitudinal momentum distribution of the partons in the hadron. By measuring precisely the exclusive channels, like the elastic diffractive vector meson production or deeply virtual Compton scattering one will be able to extract the three-dimensional distributions of the partons inside the hadron. The measurements of the momentum transfer dependence of such processes will allow, through the Fourier transform, to map the spatial transverse distributions of gluons and quarks inside the targets. Through the measurements of the exclusive diffractive dijets as well as charm, one can in principle extract the Wigner functions which will provide the information about the transverse momentum distributions of partons and spatial transverse structure at the same time. The spin dependent transverse momentum distributions could be extracted from such exclusive processes as well as from the semi-inclusive deep inelastic scattering at an EIC with polarized targets.

\section{Spin structure functions}

Electron Ion Collider with polarized beams will offer unprecedented capabilities to pin down the spin structure of the proton and will have a chance to finally resolve the long standing spin puzzle: how the total proton spin emerges from the fundamental degrees of freedom. The polarized 
EIC will extend the range in $(x, Q)$ by 1-2 orders of magnitude. It will be able to do a very precise measurement of the proton spin structure function $g_{1}$. Such measurement will allow for the precise determination of the quark contribution to the proton spin as well as the gluon contribution through the measurement of the scaling violations. Strange quark contribution is also accessible in the EIC as well as the polarized deuterons would allow for the measurement of $g_{1}$ in the neutron. By constraining the quark and gluon contributions of the proton spin and subtracting them one can constrain the parton orbital angular momentum as a component of the proton spin.

\section{Higgs production in electron-proton collider}

With the center of mass energy of 1.3/3.5 TeV the LHeC/FCC-eh machines would be able to measure the HIggs production in ep collisions for the first time. The Higgs production at ep machine proceeds through the clearly distinguishable $W W$ or $Z Z$ fusion, with the charged current process being the dominant. The Higgs production CC (NC) DIS cross section in LO QCD equal to $196(25) \mathrm{fb}$ at the LHeC energy. Higgs to $b \bar{b}$ is the dominant decay mode, with the branching ratio of $60 \%$. Uniquely, $\mathrm{CC}$ and $\mathrm{NC}$ production are distinguished and the final state, with a small pile-up permits for a clean reconstruction of a Higgs boson, which is rather centrally produced, and its decay. The detailed simulations of the Higgs production and decays at LHeC and FCCeh demonstrate huge potential as a precision Higgs factory. As an example $\mathrm{LHeC}$ can achieve about $0.5 \%$ precision on the measurement of coupling to $b \bar{b}$ and about $4 \%$ for the challenging $c \bar{c}$ mode. At FCC-ep, these uncertainties can be further reduced down to $0.2 \%$ and $1.8 \%$ for $b \bar{b}$ and $c \bar{c}$ respectively.

\section{Top and electroweak measurements}

At $\mathrm{LHeC}$ and FCC-eh the precision electroweak physics can be performed by doing measurements of the $\mathrm{NC}$ and $\mathrm{CC}$ cross sections as well as more exclusive final states such as charm or top production in CC or production of electroweak gauge bosons. The range in $Q^{2}$ reached by these machines is significantly higher than at HERA, and the $\mathrm{CC}$ and $\mathrm{NC}$ cross sections are also much larger. At the $\mathrm{LHeC}$ at the highest $Q^{2}$ scales, about $70 \%$ of the $\mathrm{NC}$ cross section is dominated by the $Z$ exchange or the $\gamma Z$ interference terms. These measurements will provide very accurate determinations of the weak neutral current couplings of the light quarks, reducing dramatically the uncertainties. The electroweak physics can be accurately tested through the measurement of the scale dependence of the weak mixing angle from below the $Z$ pole to about $1 \mathrm{TeV}$. The large cross sections and the electron polarization will allow to measure accurately the novel structure functions such as $F_{2}^{\gamma Z}$. The CC electroweak sector can be uniquely accessed at high scales over many orders of magnitude in $Q^{2}$ at the $\mathrm{LHeC}$ and at FCC-eh. This provides a very precise determination of the $W$ boson mass with an uncertainty of order $10 \mathrm{MeV}$ in electron-proton collisions at the $\mathrm{LHeC}$.

Both $\mathrm{LHeC}$ and FCC-ep would become top factories with huge potential for testing the signals of physics Beyond the Standard Model. The top quark could be produced in the $t \bar{t}$ mode or as a single top through the $\mathrm{W}$ boson radiation. The cross sections for LHeC and FCC-eh for $t \bar{t}$ production are $0.05 \mathrm{pb}$ and $1.14 \mathrm{pb}$, and for single top $1.73 \mathrm{pb}$ and $15.3 \mathrm{pb}$, respectively. One flagship 
measurement is the direct measurement of the CKM matrix element $V_{t b}$ to $1 \%$ at integrated luminosity $100 \mathrm{fb}^{-1}$, which is a determination free of any model assumptions such as on the unitarity of the CKM matrix or the number of quark generations. In addition, LHeC and FCC-eh have great potential for the anomalous top quark couplings, the left handed and right handed vector and tensor couplings. The anomalous admixtures to these couplings can be measured to an accuracy better than 5\% through top decays only. Furthermore, CKM matrix elements $V_{t s}, V_{t d}$ can be also measured to an excellent accuracy through the the analysis of $\mathrm{W}$ boson and bottom (light) quark associated production channels.

\section{Scattering on nuclear targets}

Future electron-ion machine will have a unique chance to completely transform our knowledge on the partonic structure of nuclei. At present, only fixed target data on DIS on nuclei are available, which do not provide stringent constraints on the nuclear parton distribution functions. The future electron-ion collider like EIC, LHeC or FCC-eh would dramatically improve this situation. Nuclear parton distribution functions, which are currently practically unknown at values of $x$ below 0.01 would be very well constrained, due to the high luminosity of these machines and the hugely extended kinematic regime in $x, Q^{2}$ over the present fixed target data. This will enable to provide the nuclear PDFs independently of the proton PDFs, and will provide the information on the nuclear binding including the flavour dependent shadowing effects. The diffractive parton distribution functions in nuclei have never been measured and could be extracted with a precision comparable to the projected proton diffractive parton distribution functions. The exclusive diffractive vector meson production could also be measured in the DIS on nuclear target, with the possibility of distinguishing between the coherent and incoherent cases. This will provide important information about the spatial fluctuations of the parton density in nuclei. The possibility of reaching simultaneously low $x$ region and performing scattering on nuclei would allow to test for the parton saturation phenomenon, and disentangle it from other nuclear or resummation effects. In addition, precise studies of the effects of hadronization and QCD radiation in nuclear matter could be performed by measurements of the jet and particle production with the very high precision and in a wide kinematic range.

\section{Acknowledgments}

AMS was supported by the Department of Energy Grant No. DE-SC-0002145, and the National Science centre, Poland, Grant No. 2015/17/B/ST2/01838.

\section{References}

[1] A. Accardi et al., Eur. Phys. J. A 52, no. 9, 268 (2016).

[2] J. L. Abelleira Fernandez et al. [LHeC Study Group], J. Phys. G 39, 075001 (2012).

[3] A. Abada et al. [FCC Collaboration], CERN-ACC-2018-0056. 